\title{
Influence of Au on Ge Crystallization and Its Thermoelectric Properties in a Au-induced Ge Crystallization Technique
}

\author{
S. Shanthi ${ }^{1,2,4}$ K. Faizan ${ }^{1,2}$, S. Nishino ${ }^{3}$, M. Omprakash ${ }^{2}$, T. Takeuchi ${ }^{3}$, Y. Shimura ${ }^{1,2}$, \\ Y. Hayakawa ${ }^{1,2}$, C. Muthamizhchelvan ${ }^{4}$, and H. Ikeda ${ }^{1,2, *}$ \\ ${ }^{1}$ Graduate School of Science and Technology, Shizuoka University, Japan \\ ${ }^{2}$ Research Institute of Electronics, Shizuoka University, Japan \\ ${ }^{3}$ Energy Materials Laboratory, Toyota Technological Institute, Japan \\ ${ }^{4}$ Department of Nanotechnology, SRM University, India \\ shanthiselvaraj43@gmail.com, khan.faizan.16@shizuoka.ac.jp \\ s_nishino@toyota-ti.ac.jp, omprakashmuthusamy@gmail.com \\ t_takeuchi@toyota-ti.ac.jp, shimura.yohsuke@shizuoka.ac.jp \\ hayakawa.yasuhiro@shizuoka.ac.jp,selvancm@gmail.com \\ *ikeda.hiroya@shizuoka.ac.jp
}

\begin{abstract}
Poly-crystalline $\mathrm{Ge}$ (pc-Ge) thin films were prepared on a $\mathrm{SiO}_{2} / \mathrm{Si}$ substrate using Au-induced crystallization (GIC) of amorphous $\mathrm{Ge}(\mathrm{a}-\mathrm{Ge}$ ) with an annealing temperature around the eutectic point of Au-Ge alloy system $\left(361^{\circ} \mathrm{C}\right)$ in order to shorten the annealing time. Bilayer thin films of $\mathrm{Au}(20 \mathrm{~nm}) / \mathrm{a}-\mathrm{Ge}(100 \mathrm{~nm})$ were used as a precursor material and annealed at 300,400, and $500^{\circ} \mathrm{C}$ for $60 \mathrm{~min}$, which successfully leads to the formation of pc-Ge layers. Characterizing the prepared Ge layers, the crystallographic properties indicated that the metal catalyst Au plays a notable role of enhancing both the crystallization and the island formation of Ge layers. It was also shown that the pc-Ge hardly contains Au atoms. Therefore, the Seebeck coefficient was hardly influenced by $\mathrm{Au}$ atoms since they do not act as a carrier source. In addition, the thermal conductivity of the pc-Ge film prepared by the GIC method was higher than that formed without Au, which is not due to the Au catalyst itself but due to the crystallinity of Ge film enhanced by the Au atoms.
\end{abstract}

INDEXING TERMS/KEYWORDS: Group-IV semiconductors, Au induced crystallization, thermal conductivity seebeck coefficient.

Language: English

Date of Publication: 30-06-2018

DOI: $10.24297 / j a p . v 14 i 2.7421$

ISSN: 2347-3487

Volume: 14 Issue: 2

Journal: Journal of Advances in Physics

Website: https://cirworld.com

This work is licensed under a Creative Commons Attribution 4.0 International License. 


\section{INTRODUCTION}

In recent years, thermoelectric conversion techniques have gained attention due to their potential for overcoming the energy and environmental issues. However, the thermoelectric power generator does not have sufficient conversion efficiency for practical use. The efficiency of power generator monotonously increases with increasing the thermoelectric figure-of-merit that is proportional to electrical conductivity and square of Seebeck coefficient and inversely proportional to thermal conductivity. For enhancing the performance of thermoelectric power generator, the introduction of nanostructures, such as nanowires, has been expected and widely investigated since the carrier confinement effect can rise the Seebeck coefficient and the phonon confinement effect leads to the reduction of the thermal conductivity[1-9]. As a thermoelectric nanostructure, group-IV semiconductors, $\mathrm{Si}, \mathrm{Ge}$, and $\mathrm{SiGe}$ are focused to be well defined by ultra-large scale integrated-circuit technology and compatible to Si-based devices. Thin Si-, Ge-, and SiGe-on-insulator (SOI, GOI, and SGOI) layers are required to enable the formation of patterned nanostructures on a substrate.

We have focused on $\mathrm{Ge}$ as a thermoelectric material since crystalline $\mathrm{Ge}$ (c-Ge) has higher electron and hole mobility and lower thermal conductivity compared with crystalline Si (c-Si)[10]. For the fabrication of thin GOI layer, the metal-induced crystallization (MIC) with catalytic metals, such as $\mathrm{Au}, \mathrm{Al}$ and $\mathrm{Ni}$, is a distinguished technique of conversion of amorphous Ge (a-Ge) into c-Ge[10-15]. Particularly, the MIC using Au catalyst is gaining interest, with respect to the lower temperature process and the crystalline quality[16]. Most of reports related to the Au-induced crystallization (GIC) technique aim to fabricate a single-crystalline GOI (sc-GOI) layer, and therefore, they use low annealing temperature below $300^{\circ} \mathrm{C}$ and long annealing time of 50-150 h[17-20]. In order to lower the thermal conductivity, however, poly-crystalline GOI (pc-GOI) is preferable rather than scGOI since the mean-free-path of phonons is expected to be decreased due to an increase in scattering at the grain boundary, which leads to suppression of thermal conduction.

In this paper, we aim to fabricate pc-GOI layers by GIC with a shorter annealing time. For this purpose, we choose the annealing temperature around the eutectic point of the liquid phase in the Au-Ge alloy system $\left(361{ }^{\circ} \mathrm{C}\right.$ at $\mathrm{Au} / \mathrm{Ge}$ of $\left.28 \% / 72 \%\right)$, which is much smaller than melting points of $\mathrm{Au}\left(1064{ }^{\circ} \mathrm{C}\right)$ and $\mathrm{Ge}\left(938^{\circ} \mathrm{C}\right)[21]$. The crystallographic and thermoelectric properties of the GIC-grown GOI thin film were characterized, and the influence of $\mathrm{Au}$ atoms on the crystallization and the thermoelectric characteristics is demonstrated.

\section{MATERIALS AND METHODS}

The sample preparation procedure is schematically illustrated in Fig. 1. Commercial $\mathrm{n}$-type Si substrates covered with 103-nm-thick $\mathrm{SiO}_{2}$ were cut to $1 \mathrm{~cm} \times 1 \mathrm{~cm}$. After pre-cleaning with piranha solution, a Ge layer with a thickness of $100 \mathrm{~nm}$ was deposited by vacuum evaporation in a vacuum chamber with a base pressure of $6.0 \times 10^{-4} \mathrm{~Pa}$ at room temperature. Then, a 20-nm-thick Au layer was deposited on the Ge film using Au sputtering at room temperature. Finally, the samples were annealed at 300,400 and $500{ }^{\circ} \mathrm{C}$ for 60 min in a $\mathrm{N}_{2}$ ambient without using any capping layers. After the completion of the annealing process, the residual $\mathrm{Au}$ grains were etched using aqua regia. With the aim of clarifying the Au influence, the Ge layers without a $\mathrm{Au}$ layer were also prepared under the same deposition/annealing conditions.

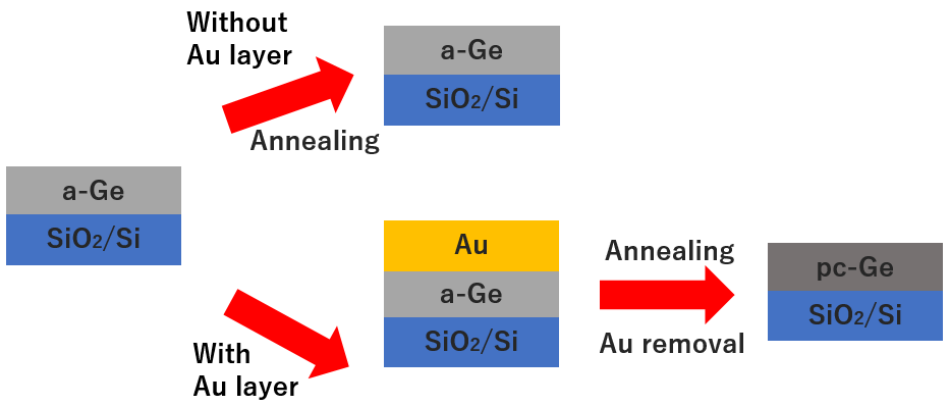

Fig. 1. Schematic of Au-induced crystallization process of $\mathrm{Ge}$ film on $\mathrm{SiO}_{2} / \mathrm{Si}$ substrate. As a reference, the Ge layers without a Au layer were also prepared under the same annealing conditions. 
The prepared layers were examined using X-ray diffractometer, XRD (RINT-2200, CuKa radiation, Rigaku) with $0.04^{\circ} \mathrm{sec}^{-1}$ scan rate in the $2 \theta$ range from $20^{\circ}$ to $80^{\circ}$, Raman scattering spectroscopy (NRS-7100, JASCO) with a laser excitation wavelength of $532 \mathrm{~nm}$ (spot size $1 \mu \mathrm{m}$ ), field-emission scanning electron microscopy, FE-SEM (JSM 6335F, JEOL), and electron probe micro analysis, EPMA (JXA-8530, JEOL). The Seebeck coefficient was measured at room temperature after forming Pt electrodes, using a conventional method[22,23]. Using picosecond time-domain thermoreflectance (TDTR)[24], thermal conductivity was determined at room temperature. Before measurement, a Mo layer with a thickness of $100 \mathrm{~nm}$ was deposited on the sample surface for the front-heating front-detection method of TDTR. In evaluating the thermal conductivity from the thermal effusivity, the specific heat capacity $323 \mathrm{Jkg}^{-1} \mathrm{~K}^{-1}$ and the density $5324 \mathrm{kgm}^{-3}$ were used for Ge.

\section{RESULTS AND DISCUSSION}

Figure 2 indicates FE-SEM images of the Ge layer surfaces after annealing at 300,400 and $500{ }^{\circ} \mathrm{C}$ for with and without a Au layer. While the Ge layers without Au catalyst seem to have a smooth surface as shown in Figs. 2(a-c), the Ge surfaces formed by the GIC method show bright domains in the SEM images of Figs. 2(d-f). Fig. 3 is EPMA images (top), elemental line profile (middle), and Ge distribution (bottom) of the GIC-formed Ge films. In the elemental line profile, obtained at a yellow line in the EPMA image, Si and O signals are observed all over the prepared layer and there is no significant difference in their amounts among the samples. These signals originate from the $\mathrm{SiO}_{2} / \mathrm{Si}$ substrate. The Ge distribution mostly coincide with the bright domains observed in the EPMA image. The elemental line profile in the $500^{\circ} \mathrm{C}$-annealed sample clearly indicates that the $\mathrm{Ge}$ intensity increases at the bright domain while the Au intensity is significantly observed at the dark regions. These findings strongly suggest that the bright domains in the SEM images of Figs. $2(d-f)$ correspond to Ge islands and contain few Au atoms.
Annealing conditions
$300^{\circ} \mathrm{C}$
$400{ }^{\circ} \mathrm{C}$
$500{ }^{\circ} \mathrm{C}$
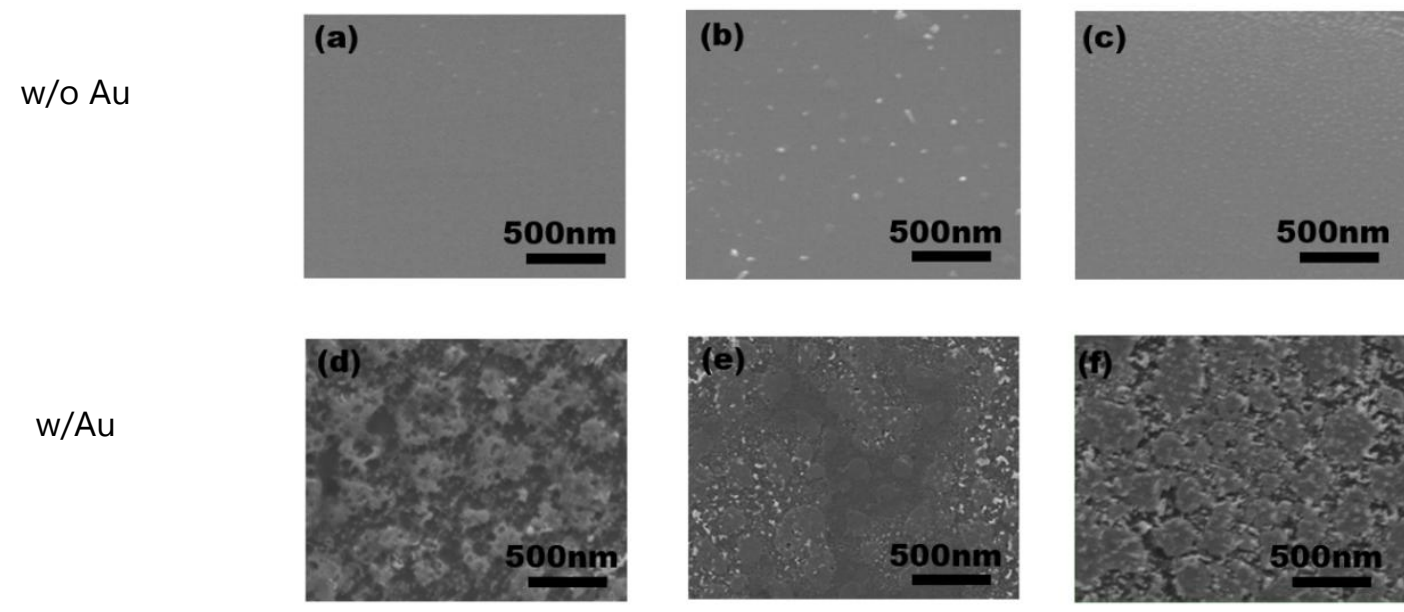

Fig. 2. FE-SEM images of Ge film annealed at 300,400 and $500^{\circ} \mathrm{C}$ with and without Au catalyst.

In addition, from the line profile of 300 and $400{ }^{\circ} \mathrm{C}$ annealed layers, a thin Ge film is found to lie between the $\mathrm{Ge}$ islands. On the other hand, the thin Ge film vanishes and the Ge islands are isolated after annealing at 500 ${ }^{\circ} \mathrm{C}$. Therefore, the Ge-island agglomerates each other and its size progresses as the annealing temperature increases. These results indicate that the Au catalyst enhances the island formation of the Ge film, which is considered due to lowering the surface energy of the sample by the Au layer.

XRD profiles of the Ge layers annealed at 300,400 and $500^{\circ} \mathrm{C}$ for with and without a Au layer are shown in Fig. 4. It is found from Fig. 4(a) that the significant peak is hardly observed in these profiles except for the Si (200) peak at $\sim 33.4^{\circ}$ originating from the Si substrate. This result indicates that the Ge layer remains amorphous, resulting in the flat surface observed in SEM images (Fig. 2). Therefore, no significant change happens without $\mathrm{Au}$ at these annealing temperatures. In contrast, in the XRD profiles of the Ge layers formed by GIC (Fig. 4(b)), 
a Ge (111) peak at $27.4^{\circ}$ can be clearly observed even for the $300^{\circ} \mathrm{C}$-annealed Ge layer. Hence, it can be said that the Au catalyst plays a role of enhancing the crystallization of Ge film. The Ge (111) peak becomes more significant at annealing temperature above $400^{\circ} \mathrm{C}$. Considering that these annealing temperatures are higher than the eutectic temperature of the Au-Ge alloy system, the crystallization of Ge seems to be enhanced due to the existence of the liquid-phase Ge. However, as shown in the EPMA results, the Ge island contains a much less amount of $\mathrm{Au}$ atoms than the Au concentration at the eutectic point, 28\% [21]. Therefore, Au atoms may act exactly as a catalyst rather than the effect of eutectic point. For example, they could provide a nucleation site for Ge crystallization. Anyway, the GIC of Ge successfully occurs by 60 min annealing.

Annealing

conditions

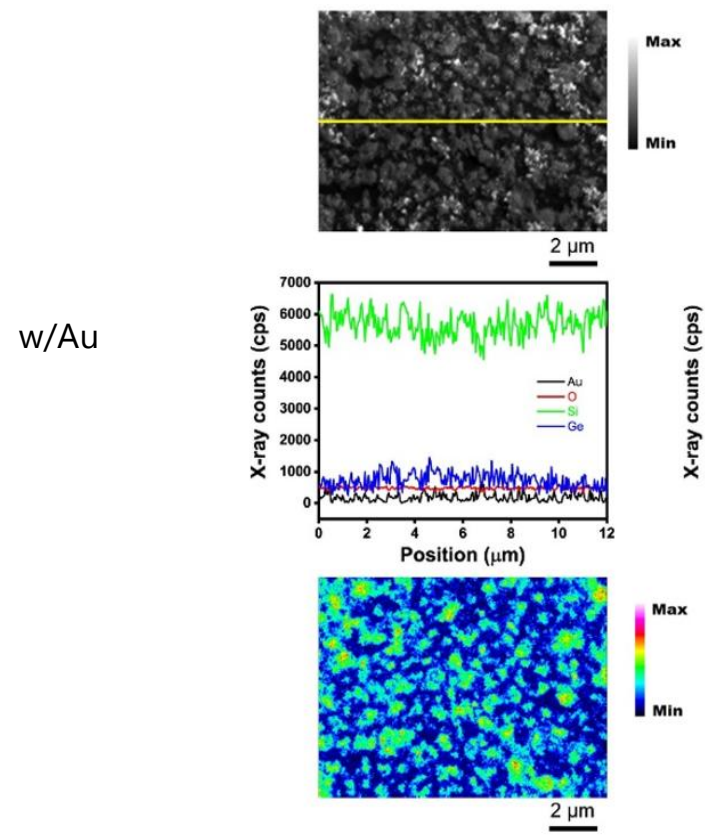

$300^{\circ} \mathrm{C}$

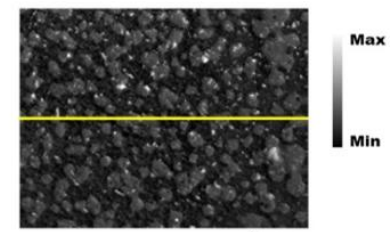

$\underline{2 \mu \mathrm{m}}$
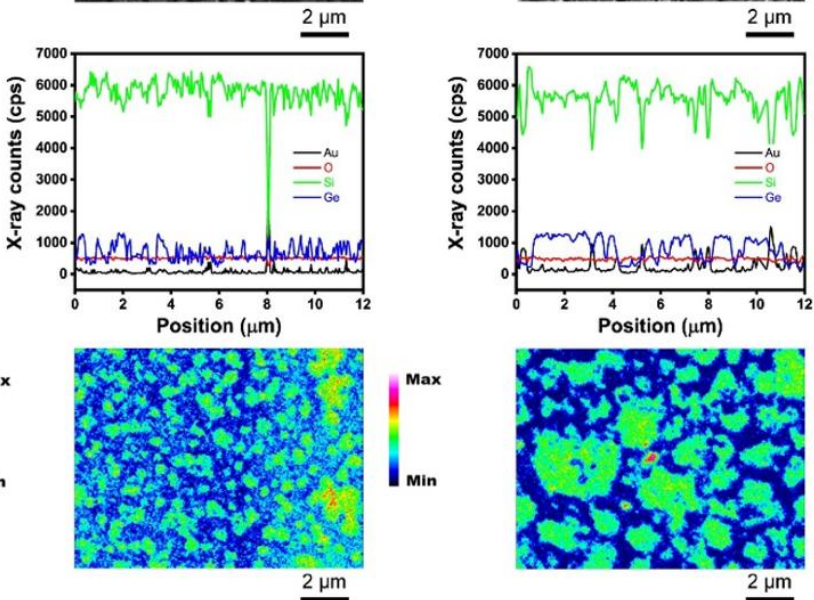

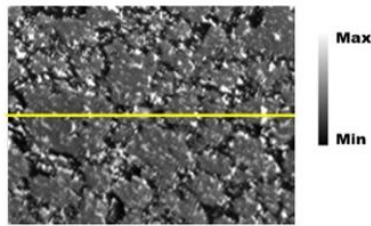

$2 \mu \mathrm{m}$

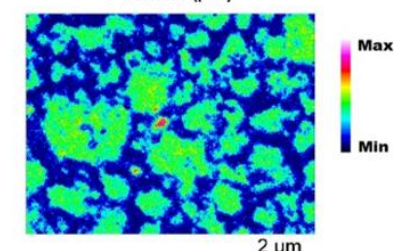

Fig. 3. EPMA images (top), elemental line profile (middle), and Ge distribution (bottom) of GIC-formed Ge films. The line profile corresponds to the yellow line in the EPMA image. The color bar in the bottom images indicates the intensity of $\mathrm{Ge}$-atom signals.
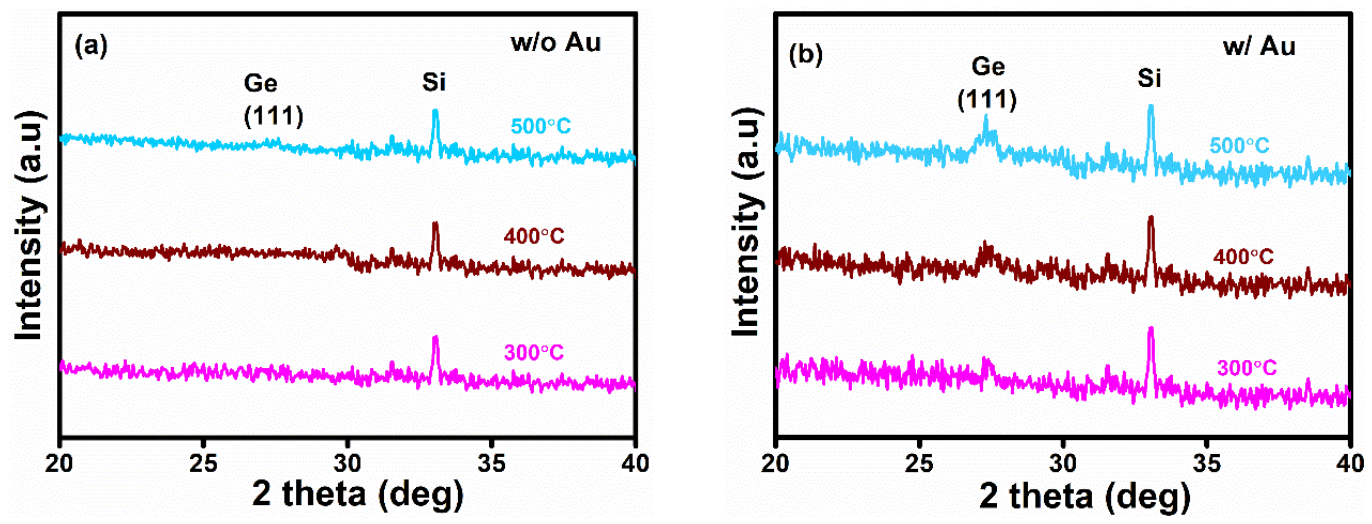

Fig. 4. XRD profiles of annealed Ge films (a) without and (b) with Au catalyst.

Figure 5 shows Raman spectra obtained from the Ge layers annealed at 300,400 and $500{ }^{\circ} \mathrm{C}$ for with and without a Au layer. For comparison, the Raman spectrum of the as-deposited Ge film was also taken (data not shown here). It was confirmed that the as-deposited $\mathrm{Ge}$ has a broad peak at $284 \mathrm{~cm}^{-1}$ corresponding to $\mathrm{Ge}-\mathrm{Ge}$ bonds in a-Ge[25]. The a-Ge peak is observed in the prepared Ge layers without $\mathrm{Au}$ catalyst even after 
annealing at $500{ }^{\circ} \mathrm{C}$, as shown in Fig. 5(a). For the GIC-formed Ge layer, in contrast, a sharp peak of Ge-Ge bond at $297 \mathrm{~cm}^{-1}$ is clearly observed, as shown in Fig. 5(b), which is assigned to c-Ge [25]. These findings indicate that the Au catalyst makes the Ge film crystalized even at $300{ }^{\circ} \mathrm{C}$ while the Ge film without Au is dominantly amorphous after annealing at $500^{\circ} \mathrm{C}$, which is consistent with the results of SEM and XRD. The GeGe peak position observed in the GIC-formed Ge film is slightly lower than $300 \mathrm{~cm}^{-1}$ in bulk c-Ge. This would be due to amorphous and nano-crystalline Ge present in the layer[26]. Moreover, it was confirmed that Ge-Au component is below the detection limit in Raman spectra. Therefore, it cannot be distinguished if residual Au observed by EPMA makes Ge-Au bonds as a substitutional Au atom.
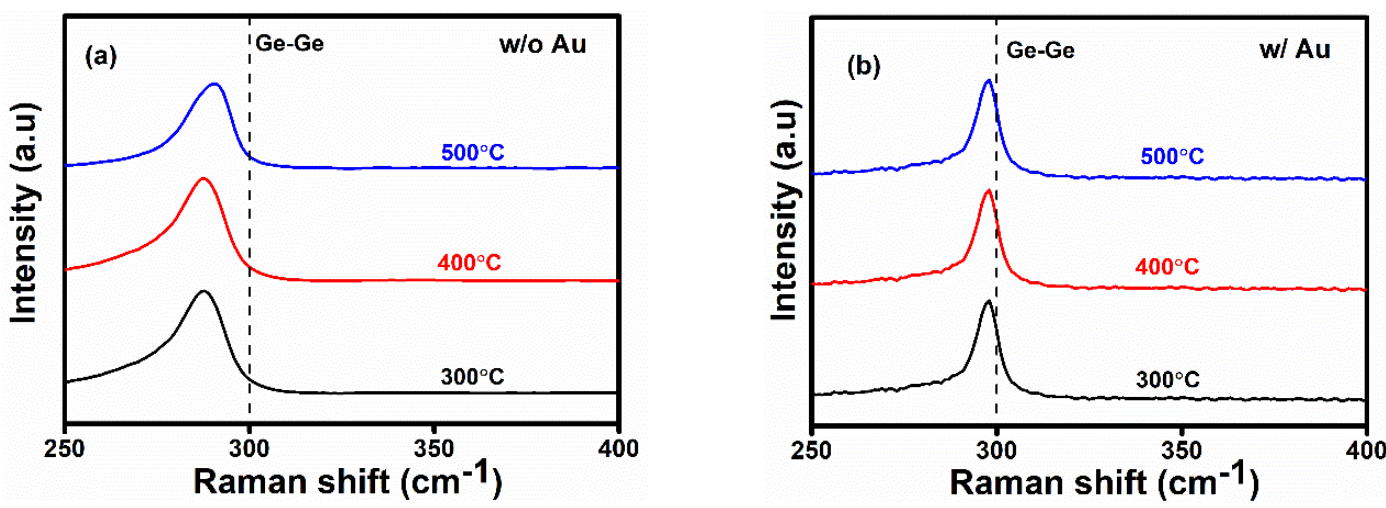

Fig. 5. Raman spectra of annealed Ge films (a) without and (b) with Au catalyst.

The Seebeck coefficient of the Ge layer with and without Au catalyst are shown in Fig. 6(a), as a function of annealing temperature. The data of the as-deposited $\mathrm{Ge}$ layer are also indicated in these graphs for comparison. The Seebeck coefficient for the Ge layer with (or without) Au annealed at $400{ }^{\circ} \mathrm{C}$ could not be measured owing to its high electric resistance, which indicates a small amount of carriers in the Ge layer. Although the measured Seebeck coefficients scatter in Fig. 6(a), there is not significant difference between with and without Au catalyst.
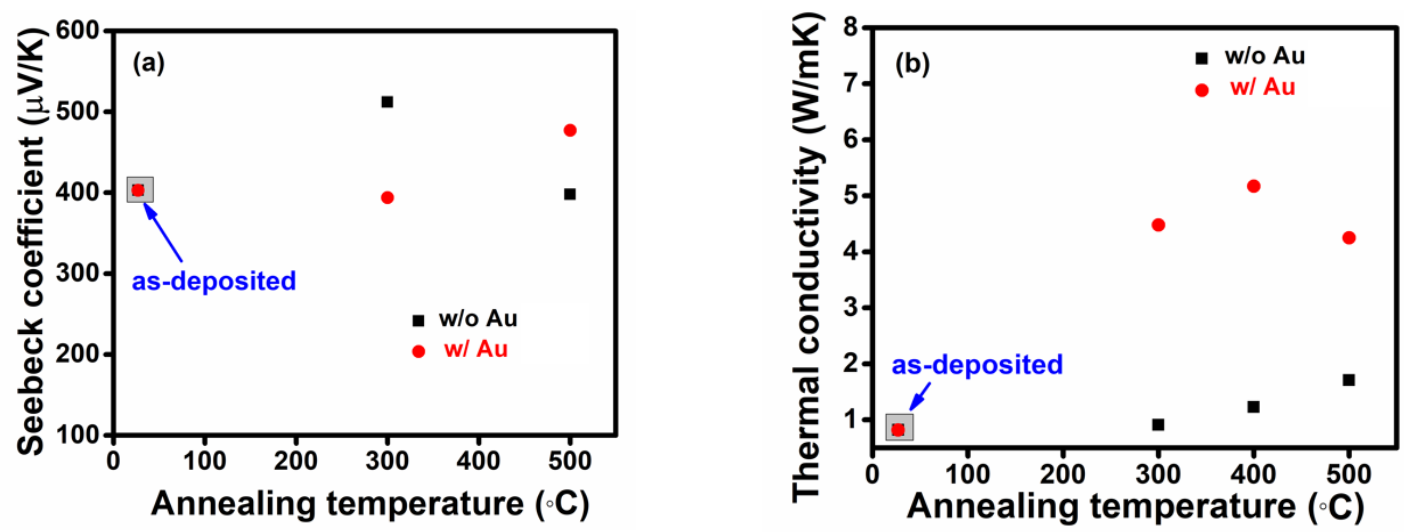

Fig. 6. (a) Seebeck coefficient and (b) thermal conductivity of Ge film with and without Au catalyst, as a function of annealing temperature.

As described above, it could not be confirmed that Au makes Ge-Au bonds in the Ge film, however, even if there is Ge-Au bond, the impurity level formed in Ge energy bandgap is too deep to act as acceptor at room temperature[27]. Therefore, the Seebeck coefficient is not influenced by Au atoms. It is reported that Au atoms remain in the Ge layer and work as a carrier source in the case using repeated $\mathrm{Au} / \mathrm{Ge}$ bilayers as a precursor material[28]. In this case, the carrier concentration seems difficult to be controlled because the carrier concentration is automatically above $7 \times 10^{18} \mathrm{~cm}^{-3}$. In our preparation procedure, the carrier concentration can be controlled by the additional doping process since there is few Au atom in the prepared GOI layer. Figure $6(b)$ is the thermal conductivity of the Ge layer with and without Au catalyst as a function of annealing 
temperature. The measured value of the as-deposited Ge layer is $0.82 \mathrm{Wm}^{-1} \mathrm{~K}^{-1}$, which is close to the reported value[29]. It is found that the crystallized Ge film formed by GIC has larger thermal conductivity than the Ge film formed without $\mathrm{Au}$. The increase in the thermal conductivity of the MIC-formed Ge film is considered to originate from the crystallization of the Ge film, taking account of the result that the Ge layer contains few Au atoms and the fact that the thermal conductivity in a crystal material is usually larger than that in an amorphous material.

\section{CONCLUSIONS}

We investigated the pc-GOI layer grown by a GIC method with a short annealing time and clarified the influence of Au catalyst on the crystallization and the thermoelectric characteristics. After annealing only for 60 min, the pc-GOI layer was successfully obtained by tuning the annealing temperature around the eutectic temperature of the Au-Ge alloy system. The SEM, EPMA, XRD, and Raman scattering spectroscopy results indicate that the Au layer plays a role of enhancing the crystallization and island-formation of the Ge film. As for the thermoelectric characteristics, the Seebeck coefficient is hardly influenced by a small amount of residual $\mathrm{Au}$ atoms while the thermal conductivity is increased in the GIC-formed Ge layer. This is caused by the crystallinity of the Ge film because the crystallographic properties indicated that there are few Au atoms in the prepared Ge layer.

\section{CONFLICTS OF INTEREST}

The authors declare no conflicts of interest.

\section{ACKNOWLEDGMENTS}

This work was financially supported in part by a JST CREST Grant Number JPMJCR15Q7, Japan.

\section{REFERENCES}

[1] L.D. Hicks, T.C. Harman, X. Sun, and M. S. Dresselhaus, Phys. Rev. B 47, (1993),12727.

[2] L.D. Hicks, and M. S. Dresselhaus, Phys. Rev. B 47, (1993), 16631.

[3] M. V. Simkin, and G. D. Mahan, Phys. Rev. Lett. 84, (2000), 927.

[4] N. Neophytou, and H. Kosina, Phys. Rev. B 83, (2011), 245305.

[5] Z. G. Chen, G. Han, L. Yang, L. Cheng, and J. Zou, Prog. Nat. Sci. Mater. Intl. 22, (2012), 535.

[6] X. Zhang, and L.D. Zhao, J. Materiomics, 1, (2015), 92.

[7] C. Gayner, and K. K. Kar, Prog. Mater. Sci. 83, (2016), 330.

[8] P. Pichanusakorn, and P. Bandaru, Mater. Sci. Engg. R 67, (2010), 19.

[9] Y. Wun, S. W. Finefrock, and H. Yang, Nano Energy, 1, (2012), 651.

[10] Q. Li, C. Chen, Z. Chen, Z. Jiao, M. Wu, C. H. Shek, C. M. L. Wu, and J. K. L. Lai, Inorg. Chem. 51, (2012), 8473.

[11] K. Toko, K. Nakazawa, N. Saitoh, N. Yoshizawa, and T. Suemasu, Cryst. Grow. Des. 15, (2015), 1535.

[12] M. Kurosawa, Y. Tsumura, T. Sadoh, and M. Miyao, Jpn. J. Appl. Phys. 48, (2009), $03 B 002$.

[13] H. Kanno, T. Aoki, K. A. Kenjo,T. Sadoh, and M. Miyao, Mater. Sci. in Semi. Pro. 8, (2005), 79.

[14] J. H. Park, T. Suzuki, M. Kurosawa, M. Miyao, and T. Sadoh, Appl. Phys. Lett. 103, (2013), 082102. 
[15] Y. C. Her, J. H. Chen, M. H. Tsai, and W. T. Tu, J. Appl. Phys. 106, (2009), 023530.

[16] T. Sugiyama, N. Mishiba, M. Kamiko, and K. Kyuno, Appl. Phys. Exp. 9, (2016), 095501.

[17] M. H. Park, H. J. Kim, Y. J. Kim, T. Moon, and C. S. Hwang, Appl. Phys. Lett. 104, (2014), 072901.

[18] T. Sadoh, J. H. Park, R. Aoki, and M. Miyao, Thi. Sol. Fil. 602, (2016), 3.

[19] H. Okamoto, K. Kudo, T. Nomitsu, R. Mochii, K. Moto, K. Takakura, and I. Tsunoda, Jpn. J. Appl. Phys. 55, (2016), 04EJ10.

[20] T. Sadoh, J. H. Park, R. Aoki, and M.Miyao, Jpn. J. Appl. Phys. 56, (2017), 129206.

[21] H. Okamoto, and T. B. Massalski, Bull. Alloy Phase Diagrams, 5, (1984), 601.

[22] F. Salleh, K. Asai, A. Ishida, and H. Ikeda, Appl. Phys. Exp. 2, (2009), 071203.

[23] H. Ikeda, and F. Salleh, Appl. Phys. Lett. 96, (2010), 012106.

[24] Y. Okamoto, J. Saeki, T. Ohtsuki, and H. Takiguchi, Appl. Phys. Exp. 1, (2008), 1170011.

[25] F. A. Ferri, J.Cryst. Sol. 358, (2012), 58.

[26] K. Sakaike, S. Higashi, H. Murakami, and S. Miyazaki, Thi. Sol. Fil. 516, (2008), 3595.

[27] S. M. Sze, Physics of Semiconductor Devices (Wiley, New Jersey, 1936) 2nd ed., p. 20.

[28] H. Takiguchi, Z. Yoshikawa, H. Miyazaki, and Y. Okamoto, J. Electron. Mater. 39, (2010), 1627.

[29] D. G. Cahill, S. K. Watson, and R. O. Pohl, Phys. Rev. B 46, (1992), 6131. 